\title{
Inhibition of glypican-3 expression via RNA interference influences the growth and invasive ability of the MHCC97-H human hepatocellular carcinoma cell line
}

\author{
JIAN RUAN $^{1 *}$, FEIYE LIU ${ }^{1 *}$, XIAOHUA CHEN ${ }^{2}$, PENG ZHAO $^{3}$, NING SU $^{1}$, \\ GUOZHU XIE $^{1}$, JIANPENG CHEN ${ }^{1}$, DAYONG ZHENG ${ }^{1}$ and RONGCHENG LUO ${ }^{1}$
}

${ }^{1}$ Department of Oncology, Nanfang Hospital, Southern Medical University; ${ }^{2}$ Department of Oncology and Hematology, Panyu Central Hospital, Guangzhou 510515, Guangdong Province; ${ }^{3}$ Department of Medical Oncology, The First Affiliated Hospital, School of Medicine, Zhejiang University, Hangzhou 310003, Zhejiang Province, P.R. China

Received February 17, 2011; Accepted April 19, 2011

DOI: $10.3892 / \mathrm{ijmm} .2011 .704$

\begin{abstract}
Glypican-3 (GPC3), a membrane-bound heparan sulfate proteoglycan, is found to be overexpressed in hepatocellular carcinoma (HCC). The purpose of the present study was to investigate the possible role of GPC3 in the development of HCC. In this study, RNA interference (RNAi) with a GPC3 small hairpin RNA (GPC3 shRNA) was used to identify the effects of GPC3 on the regulation of malignant behaviors of HCC. MHCC97-H, a highly metastatic human HCC cell line in which GPC3 mRNA and protein levels were detected as the highest among the $4 \mathrm{HCC}$ cell lines assessed in this study, and was thus selected as a cell model for in vitro and in vivo experiments. The results showed that down-regulation of GPC3 can significantly inhibit the proliferative and invasive ability of MHCC97-H. Compared with the parental HCC cells, GPC3-silenced cells exhibited attenuated capacities in developing tumors in nude mice, while the growth of tumor xenografts derived from these cells dramatically regressed. In conclusion, our results suggest that GPC3 contributes to the proliferation and metastasis of HCC and that it may be a potential molecular target for HCC treatment.
\end{abstract}

\section{Introduction}

Hepatocellular carcinoma (HCC) is a highly lethal cancer with poor prognosis. It ranks as the third cause of cancer-

Correspondence to: Dr Rongcheng Luo, Department of Oncology, Nanfang Hospital, Southern Medical University, Guangzhou 510515, Guangdong Province, P.R. China

E-mail:1fy406@yahoo.com

Dr Dayong Zheng, Department of Oncology, Nanfang Hospital, Southern Medical University, Guangzhou 510515, Guangdong Province, P.R. China

E-mail: zhengdayong@gmail.com

*Contributed equally

Key words: hepatocellular carcinoma, malignant behavior, glypican-3, RNA interference related deaths in East Asia and sub-Saharan Africa, and as the second cause of cancer-related deaths among males in China (1). The incidence of HCC is currently also increasing in the United States and Europe (2). Surgical resection remains the standard choice of treatment for patients in the early stage of HCC. However, even with radical resection, $60-70 \%$ of patients develop metastasis and recurrence within 5 years of surgery (3). Although several clinicopathological features, including a poorly differentiated phenotype, large-sized tumor, and portal venous invasion have been found to contribute to the poor prognosis in HCC patients before operation, the underlying molecular mechanisms of the development of $\mathrm{HCC}$ remain unclear. Thus, it is urgent to study the pathogenesis of HCC.

Glypican-3 (GPC3) was originally isolated as a developmentally regulated transcript from the rat intestine, and was later identified as a GPI (glycosyl phosphatidyl inositol)-linked heparan sulfate proteoglycan with a wider expression (4). In vivo, it was found to be predominantly expressed in the mesoderm and mesoderm-derived tissues, and too much lesser extent in endodermal- and ectodermal-derived tissues (5). Loss-of-function mutations in GPC3 cause the SimsonGolabi-Behmel syndrome (SGBS), which is characterized by pre- and postnatal overgrowth (6). GPC3 is released from the cell surface by a lipase called Notum, whose functions may regulate the signaling of Wnts, Hedgehogs, fibroblast growth factors, and bone morphogenetic proteins pathways, and are involved in the migration, proliferation, and modulation of cell survival in several tissues (6-9). However, GPC3 functions in a tissue- and time-specific pattern. In some tissues, GPC3 acts as a tumor suppressor, whereas in others, it serves as an oncofetal gene $(10,11)$. Previous studies have confirmed the overexpression of GPC3 in HCC (12), but the function of up-regulated GPC3 in HCC remains unclear.

We herein used RNAi by stably expressing GPC3 small hairpin RNAs (GPC3-shRNA) to analyze the roles of GPC3 in the malignant behaviors of HCC cells. Our results showed that silencing of GPC3 using GPC3-shRNA transfection reduced the cell proliferation and invasion activity in vitro and the tumorigenicity in nude mice, indicating a positive 
Table I. Oligonucleotide sequences of GPC3-specific shRNA.

\begin{tabular}{llc}
\hline Name & \multicolumn{1}{c}{ Sense/antisense strands of shRNA } & Target nucleotide sites \\
\hline MHCC-97H/GPC3-shRNA1 & 5'-TGGCTCTGAATCTTGGAATTGATTCAAGAG & $890-910$ \\
& ATCAATTCCAAGATCAGAGCCTTTTTC-3' & \\
& 5'-TCGAGAAAAAAGGCTCTGAATCTTGGAATT & \\
MHCC-97H/GPC3-shRNA2 & GATCTCTTGAATCAATTCCAAGATTCAGAGCCA-3' & \\
& 5'-TGCCGAATGCTCACCAGAATGTTTCAAGAG & $955-975$ \\
& AACATTCTGGTGAGCATTCGGCTTTTTC-3' & \\
MHCC-97H/GPC3-shRNA3 & 5'-TCGAGAAAAAAGCCGAATGCTCACCAGAAT & \\
& GTTCTCTTGAAACATTCTGGTGAGCATTCGGCA-3' & $995-1015$ \\
& 5'-TGGGACTGATGATGGTTAAACCTTCAAGA & \\
& GAGGTTTAACCATCATCAGTCCCTTTTTTC-3' & \\
MHCC-97H/GPC3-shRNA4 & 5'-TCGAGAAAAAAGGGACTGATGATGGTTAAA & \\
& CCTCTCTTGAAGGTTTAACCATCATCAGTCCCA-3' & \\
& 5'-TGGCACTTCAAATATCCTATCTTTCAAGAGA & \\
& AGATAGGATATTTGAAGTGCCTTTTTTC-3' & \\
& 5'-TCGAGAAAAAAGGCACTTCAAATATCCTAT & \\
& CTTCTCTTGAAAGATAGGATATTTGAAGTGCCA-3' & \\
\hline
\end{tabular}

association between GPC3 expression and the carcinogenesis and metastasis of HCC.

\section{Materials and methods}

Cell culture and plasmid transfection. The human hepatoma cell lines HepG2, SMMC-7721, HCCL-M6 and MHCC97-H were grown in DMEM (Gibco-BRL) supplemented with $10 \% \mathrm{FCS}$ in a $5 \% \mathrm{CO}_{2}$ humidified atmosphere at $37^{\circ} \mathrm{C}$. GPC3 mRNA and protein productions were examined with quantitative reverse transcription PCR (qRT-PCR) and Western blotting.

$R N A$ isolation, $q R T-P C R$. Total cellular RNA was isolated from 4 hepatoma cell lines using the TRIzol reagent (Invitrogen) according to the manufacturer's instructions and quantified using a UV spectrophotometer. RNA $(2 \mu \mathrm{g})$ was reverse transcribed using the Access reverse transcription system (Promega, Madison, WI) according to the standard protocol. In brief, reaction mixtures (total volume, $20 \mu \mathrm{l}$ ) containing $500 \mathrm{ng}$ cDNA were amplified at a final concentration of $250 \mathrm{nM}$ using $10 \mu \mathrm{l}$ 2X Brilliant SYBR-Green qPCR Master Mix kit (Stratagene). The primers were: GPC3 forward: 5'-GTTGTGAAAGGTGCTTATCT-3'; GPC3 reverse: 5'-GAA GGGTTTGAAAGTGGA-3' (GenBank: BC035972); $\beta$-actin forward: 5'-TAAGAAGCTGCTGTGCTACG-3'; $\beta$-actin reverse: 5'-GACTCGTCATACTCCTGCTT-3' (GenBank, NM_001101). The thermal cycling conditions were: $94^{\circ} \mathrm{C}$ for $5 \mathrm{~min}$ and 45 cycles at $94^{\circ} \mathrm{C}$ for $40 \mathrm{sec}$, at $58^{\circ} \mathrm{C}$ for $40 \mathrm{sec}$ and at $72^{\circ} \mathrm{C}$ for $40 \mathrm{sec}$. Experiments were performed in triplicate. Target gene and the $\beta$-actin gene were amplified in the same reaction. The relative quantitative analysis was performed by comparison of the $2^{-\Delta \Delta \mathrm{Ct}}$ values.

Western blotting. The cells were washed twice with cold phosphate-buffered saline (PBS) and then lysed on ice in
RIPA buffer [1X PBS, 1\% NP-40, 0.1\% sodium dodecylsulfate (SDS), $5 \mathrm{mM}$ EDTA, $0.5 \%$ sodium deoxycholate, and $1 \mathrm{mM}$ sodium orthovanadate] with protease inhibitors. Protein samples were resolved on a $6 \%$ SDS polyacrylamide gel, electrotransferred to polyvinylidene fluoride membranes and blocked in 5\% nonfat dry milk in Tris-buffered saline, pH 7.5 (100 mM NaCl, $50 \mathrm{mM}$ Tris, and 0.1\% Tween-20). Membranes were immunoblotted overnight at $4^{\circ} \mathrm{C}$ with the anti-GPC3 and anti- $\beta$-actin antibodies (Santa Cruz Biotechno$\operatorname{logy}$ ), followed by respective horseradish peroxidase-conjugated secondary antibodies. The detection was performed using an enhanced chemiluminescence kit (Sino-American Biotechno$\log$ ).

Construction of GPC3-targeted shRNA expression plasmids and cell transfection. Four pairs of shRNA were designed according to the GPC3 sequence (GenBank: BC035972) following the rules of Tuschl (13). DNA oligonucleotides targeting GPC3 (Table I) were synthesized and inserted into BamHI-HindIII linearized pGenesil-1 shRNA expression vector according to manufacturer's instructions. The pGenesil-1 negative control vector contains an shRNA insert that does not suppress the expression of genes expressed in humans, rats, or mice, and is provided with the pGenesil- 1 shRNA expression vector to provide a baseline for the experiments. All inserted sequences were verified by DNA sequencing.

According to the manufacturer's protocol, MHCC97-H cells were grown to 60-70\% confluency and transfected with different vectors (pGenesil-1-GPC3-shRNA1, 2, 3, 4, pGenesil1-negative) using Lipofectamine 2000 (Invitrogen). Stable transfectants, namedMHCC97-H/GPC3-shRNA1,MHCC97-H/ GPC3-shRNA2, MHCC97-H/GPC3-shRN-A3, MHCC97-H/ GPC3-shRNA4, MHCC97-H/negative-shRNA, were selected in a medium containing $400 \mu \mathrm{g} / \mathrm{ml} \mathrm{G} 418$ for 14 days and later maintained in the medium with $200 \mu \mathrm{g} / \mathrm{ml} \mathrm{G} 418$. qRT-PCR and Western blotting were applied to analyze GPC3 mRNA 
and protein levels, respectively. MHCC97-H/GPC3-shRNA1 and MHCC97-H/negative-shRNA were chosen for further comparison of biological behaviors.

In vitro cell growth assay. MHCC97-H, MHCC97-H/GPC3shRNA1 and MHCC97-H/negative-shRNA were prepared at a concentration of $1 \times 10^{4}$ cells $/ \mathrm{ml}$ and incubated for $1,2,3,4$, 5, 6 and 7 days, respectively. The 3-(4,5-dimethylthiazol-2-yl)2,5-diphenyltetrazolium bromide (MTT) assay was performed by adding $20 \mu \mathrm{l}$ of MTT (Promega) for $4 \mathrm{~h}$, removal of the supernatants and addition of $150 \mu \mathrm{l}$ of DMSO (Sigma) to each well. After $15 \mathrm{~min}$, the absorbance value (OD) of each well was measured with a microplate reader set at $490 \mathrm{~nm}$. All experiments were performed in triplicate.

Plate clone formation assay. Cells $\left(1 \times 10^{2}\right)$ were added to each well (3-cm in diameter) of 6-well culture plates, and each cell group contained three wells. After incubation at $37^{\circ} \mathrm{C}$ for 12 days, the cells were washed twice with PBS and stained with Giemsa solution. The number of colonies containing $\geq 50$ cells was counted under a microscope [plate clone formation efficiency $=$ (number of colonies/number of cells inoculated $)$ $\mathrm{x} 100 \%]$. Three individual experiments were performed.

Transwell assay. Cells stably expressing GPC3-shRNA1 or negative-shRNA and MHCC97-H were plated in 24-well transwell units with polycarbonate filters (Costar Corp., Cambridge, MA) containing $8-\mu \mathrm{m}$ pores. Cells were plated at $1.0 \times 10^{5}$ cells/well in $0.5 \mathrm{ml}$ of serum-free medium. The outer chambers were filled with $0.5 \mathrm{ml}$ of media containing $10 \%$ FBS. After $24 \mathrm{~h}$, the cells were fixed in methanol and stained with hematoxylin. The top surface of the membrane was gently scrubbed with a cotton bud, and the cells invading through the membrane filters were counted on glass slides, and images corresponding to the entire membrane surface were captured using an Olympus inverted microscope equipped with a CCD camera. Values were normalized to those of the MHCC97-H group in each experiment.

Tumorigenicity in nude mice. The experimental protocol was approved by China Institutional Ethics Review Committee for Animal Experimentation. Eight nude mice were randomly divided into experimental and control groups, with four mice in each group. GPC3-silencing cells and MHCC97-H/ negative-shRNA cells $\left(2 \times 10^{7} / \mathrm{mouse}\right)$ suspended in $0.2 \mathrm{ml}$ DMEM were each injected subcutaneously into the nude mice at the right flank. Tumor size was measured using calipers at the indicated times. The tumor volume was calculated according to the formula: volume $=$ length $\mathrm{x}\left(\mathrm{width}^{2}\right) / 2$. The animals were sacrificed on the 21st day after injection and the tumors were dissected and weighed. The tumorigenicity inhibition rate was calculated as [(tumor weight ${ }_{\text {control }}$ - tumor weight shRNA $)$ /tumor weight ${ }_{\text {control }}$ x $100 \%$.

\section{Results}

Expression of GPC3 in human hepatocellular carcinoma cell lines. GPC3 mRNA and protein levels were detected by qRT-PCR and Western blot analysis (Fig. 1) in the human hepatocellular carcinoma cell lines, HepG2, SMMC-7721,
A.
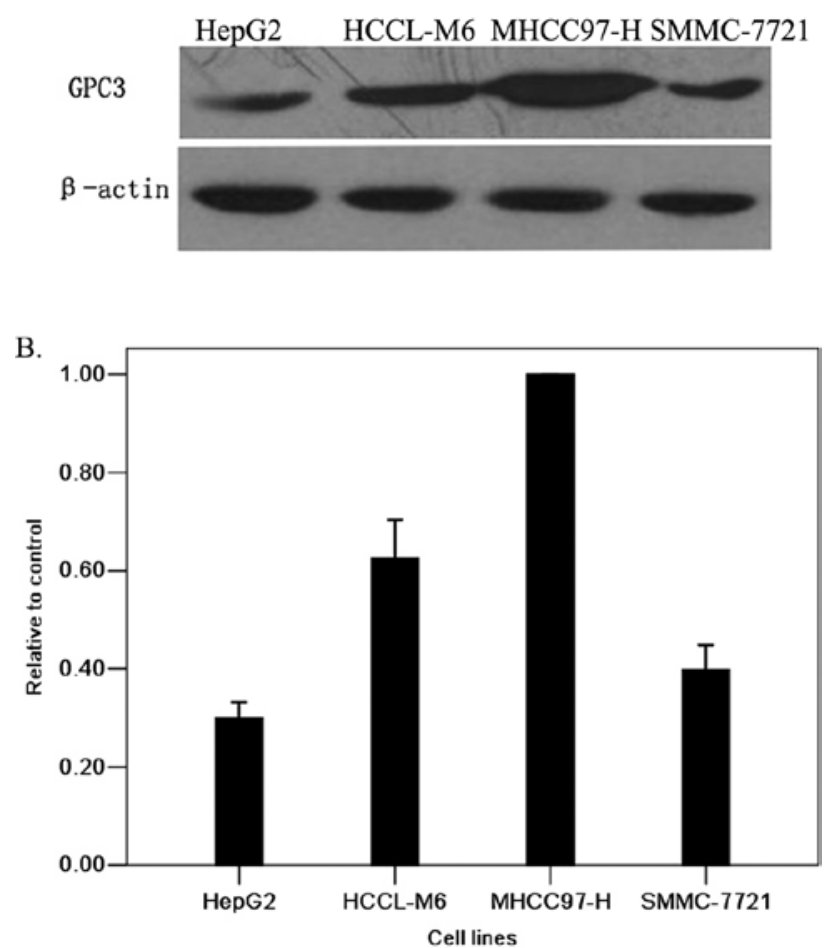

Figure 1. GPC3 expression in 4 human hepatocellular carcinoma cell lines with different metastasis potential. (A) GPC3 protein expression levels in HepG2, HCCL-M6, MHCC97-H and SMMC-7721 cell lines were determined by Western blotting. MHCC97-H showed the most remarkable increase in the expression of GPC3 protein as compared to the other cell lines. (B) Quantification of GPC3 mRNA expression in different cells related to controls HepG2, HCCL-M6, MHCC97-H, SMMC-7721 as detected by qRT-PCR. MHCC97-H expressed the highest level of GPC3 mRNA. The expression of $\beta$-actin protein and mRNA was also examined and served as the controls for sample loading. (A and B) show representative results of three independent experiments.

HCCL-M6 and MHCC97-H. Of the 4 human hepatocellular carcinoma cell lines, GPC3 was more strongly expressed in the highly metastatic MHCC97-H cells, and more weakly expressed in the non-metastatic, HepG2 cell line. Thus, the MHCC97-H cells were chosen for further investigation of the effect of specific silencing of GPC3 on malignant behaviors, such as tumor cell proliferation and invasion of hepatocellular carcinoma cells.

The vector stably expressing GPC3 shRNA effectively and specifically down-regulated GPC3 expression. The knockdown efficiencies of different GPC3-specific shRNAs in MHCC97-H cells were first evaluated using qRT-PCR. Relative GPC3 mRNA levels in individual stable transfectants were normalized against mRNA levels of an internal control gene, $\beta$-actin, performed in the same run. As shown in Fig. 2B, cells transfected with pGenesil-1-GPC3shRNA1 showed a significantly reduced transcription of GPC3 mRNA when compared with that of vector control and negative transfectants, respectively. The reduction in mRNA transcription was not detectable in cells transfected with pGenesil-1-GPC3-shRNA3 and pGenesil-1-GPC3-shRNA4. In addition, Western blot analysis (Fig. 2A) showed a remarkable reduction of GPC3 protein levels in the MHCC97-H cell lines transfected with 


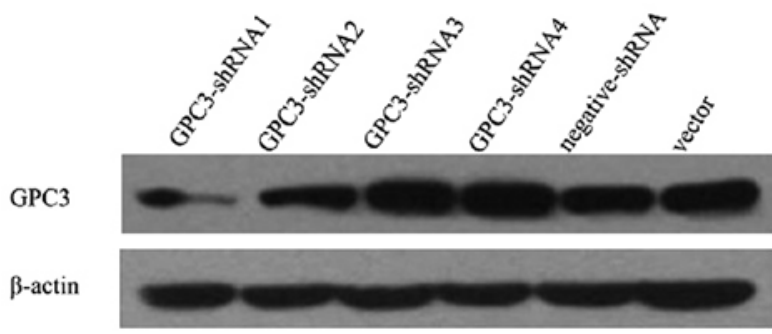

B.

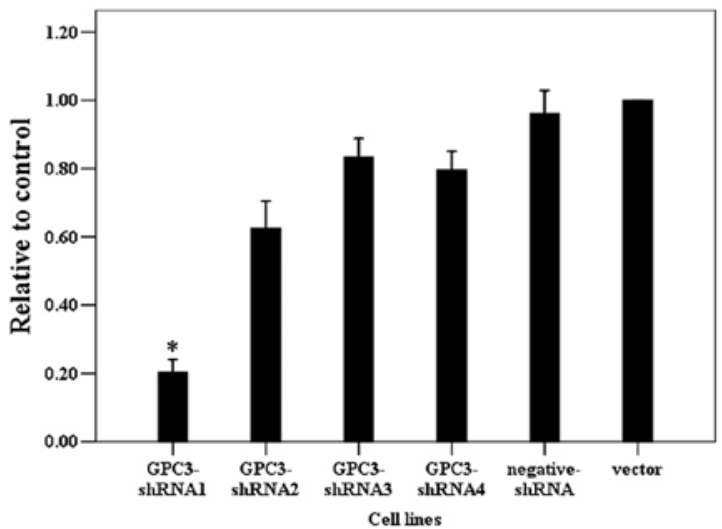

Figure 2. GPC3-specific shRNAs reduce GPC3 mRNA and protein expression in MHCC 97-H cells. (A) The levels of GPC3 protein were assessed by Western blotting. The results revealed that protein level of GPC3 in MHCC97-H cells was effectively and most significantly inhibited with GPC3-shRNA1 interference. (B) GPC3 mRNA expression in MHCC97-H/GPC3-shRNA1 was most significantly decreased compared to MHCC97-H/negative-shRNA and MHCC97-H/vector cells ( $\mathrm{P}<0.05)$. The $\beta$-actin mRNA and protein expression served as controls for sample loading.

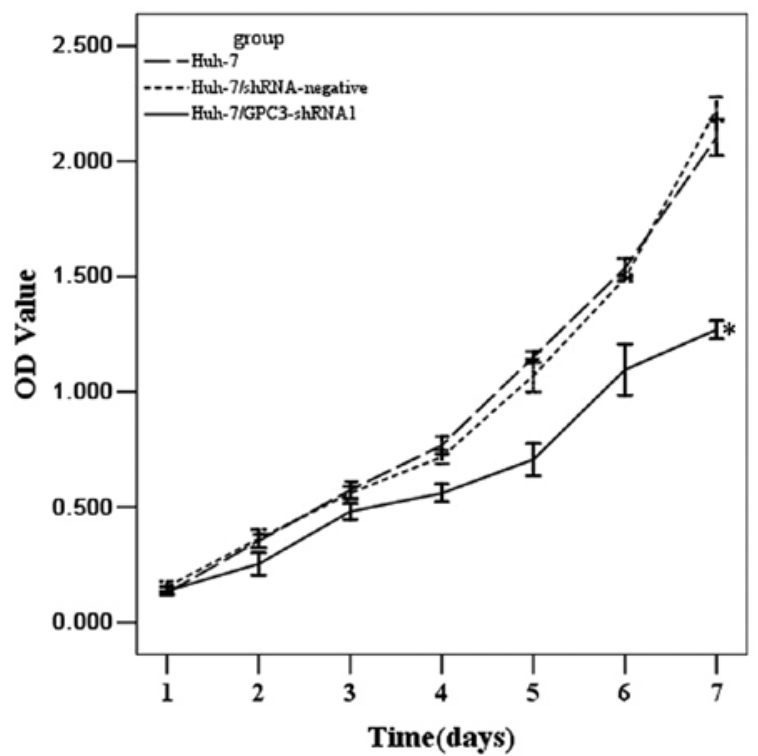

Figure 3. GPC3 gene silencing suppresses cell proliferation in vitro. Proliferation of MHCC97-H cells stably transfected with GPC3-shRNA1, negative-shRNA and vector were analyzed by the MTT assay as described earlier. The number of viable cell was assessed using the MTT assay at 1,2,3, 4, 5, 6, 7 days, respectively. Each sample was tested in triplicate and the results were reported as OD readings (mean \pm SD). MHCC97-H/GPC3-shRNA1 showed a remarkable inhibition of cell proliferation when compared with both the MHCC97-H/negative-shRNA and MHCC97-H/vector ( $\mathrm{P}<0.05)$.
A.

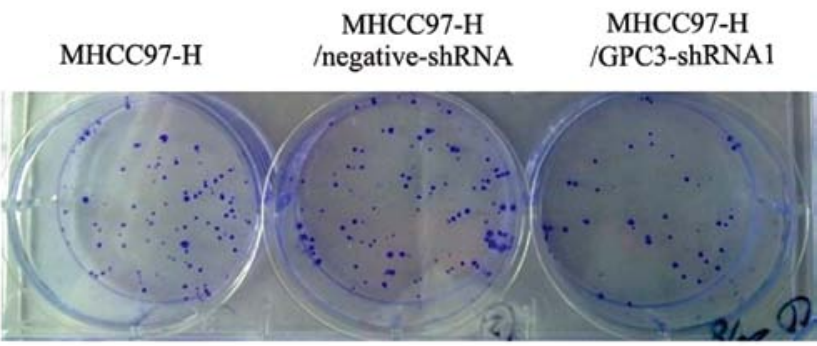

B.

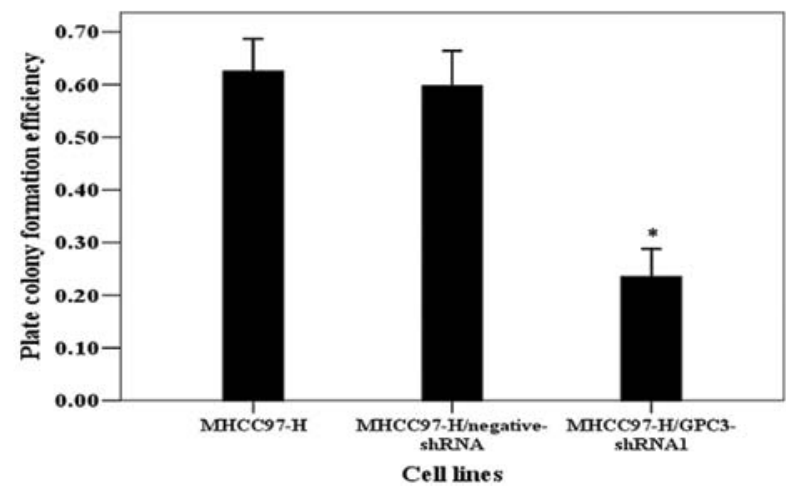

Figure 4. Effect of GPC3 silencing using shRNA on the liquid colony formation potential of MHCC97-H cells. (A) After 12 days of culture, the cells were stained with May-Giemsa and those $<1 \mathrm{~mm}$ were counted. The values represent the means \pm SD of at least three independent experiments. (B) Statistical analysis showed that the efficiency of MHCC97-H/GPC3-shRNA1 to form colonies was significantly lower than that of MHCC97-H and MHCC97-H/ negative-shRNA ( $\mathrm{P}<0.05)$.

A.

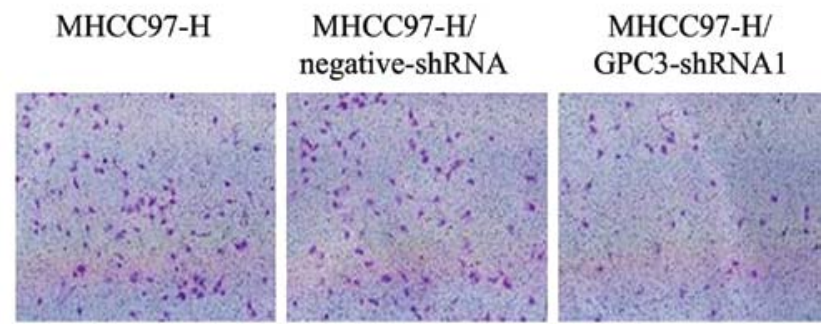

B.

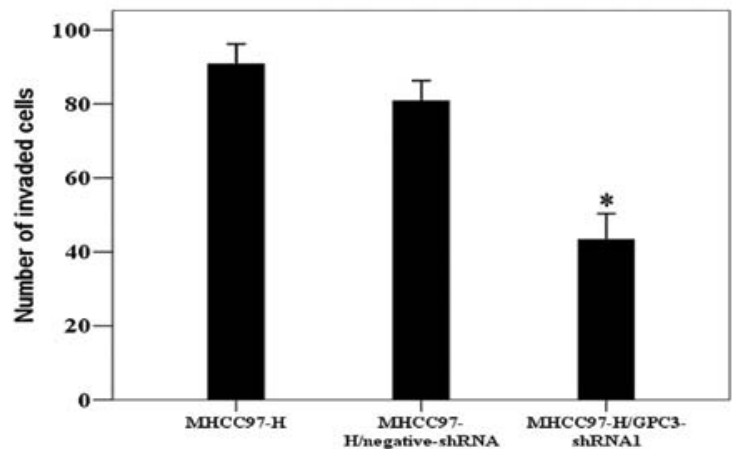

Figure 5. Effect of GPC3 knockdown on the invasive potential of hepatocellular carcinoma cells. (A) An in vitro invasion assay was carried out to compare and quantify the invasiveness of MHCC97-H, MHCC97-H/negative-shRNA, MHCC97-H/GPC3-shRNA1. Results are representative of three independent experiments and bars represent the means \pm SD. (B) GPC3 silencing cells showed a decreased invasive ability that correlated with GPC3 expression ( $\mathrm{P}<0.05$ as compared to MHCC $97-\mathrm{H} /$ negative-shRNA and MHCC97-H). 
A. MHCC97-H/negative-shRNA

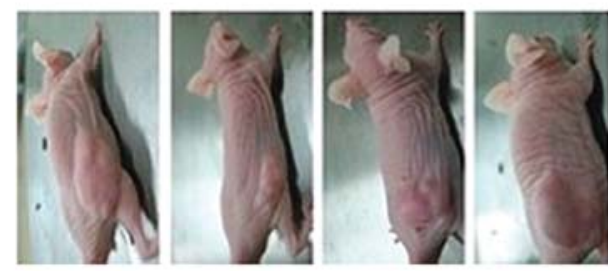

MHCC97-H/GPC3-shRNA1

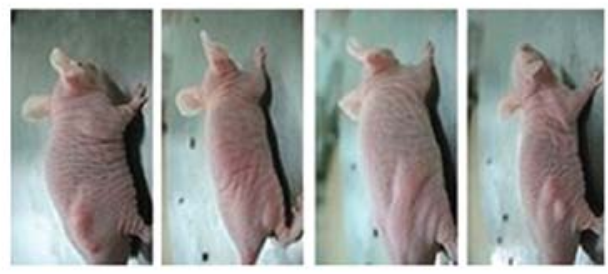

C .MHCC97-H/negative-shRNA

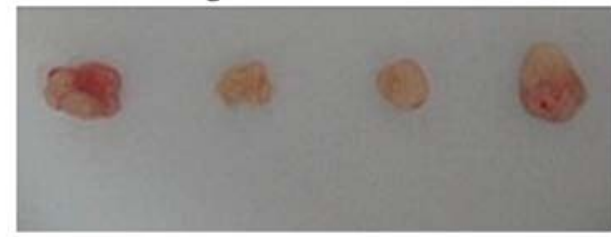

MHCC97-H/GPC3-shRNA1

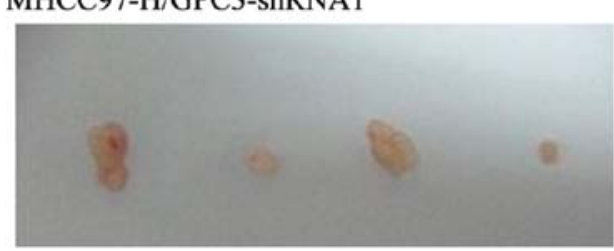

B.

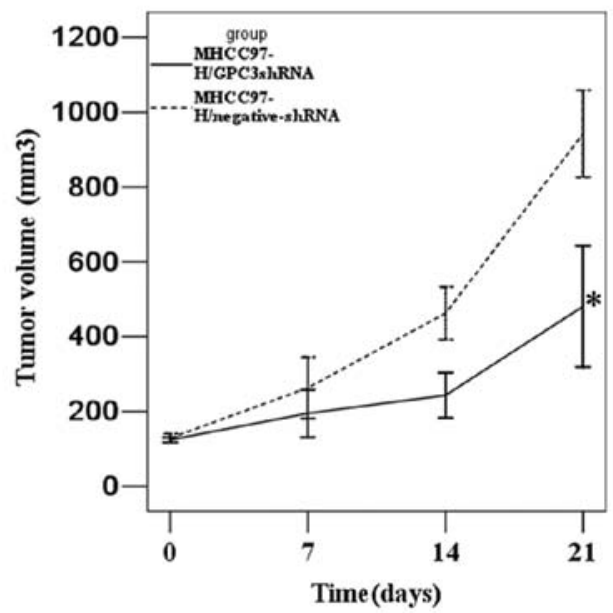

D.

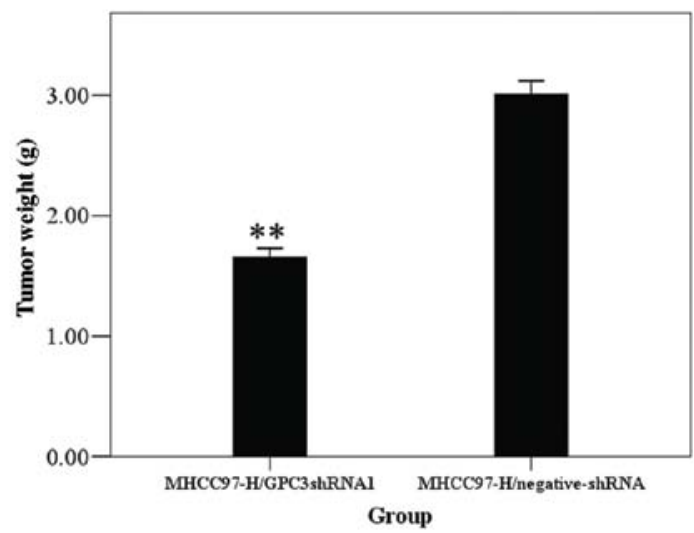

Figure 6. Knockdown of GPC3 expression in the MHCC97-H cells retards tumorigenicity in nude mice. MHCC97-H/GPC3-shRNA1 and the control cells (MHCC97-H/negative-shRNA) were injected subcutaneously into nude mice at the right flanks. (A) Tumor diameters were measured at a regular interval for up to 21 days with calipers. Results represent the means $(\mathrm{n}=4)$. (B) MHCC97-H/GPC3-shRNA1 showed a significant decrease in the growth rate, as compared to MHCC97-H/negative-shRNA ("P<0.05). (C) On the 21st days after injection and the tumors were dissected and weighed. (D). The average tumor weight ( $\mathrm{n}=4)$ of the groups MHCC97-H/GPC3-shRNA1 and MHCC97-H/negative-shRNA was $1.31 \pm 0.06$ and $2.83 \pm 0.17 \mathrm{~g}$, respectively $\left.{ }^{\left({ }^{* *}\right.} \mathrm{P}<0.01\right)$.

pGenesil-1-GPC3-shRNA1. The above results demonstrate that the GPC3 expression could be specifically and effectively down-regulated by GPC3-specific shRNA, and that different shRNA showed striking differences in silencing efficiency.

Effects of GPC3-specific shRNAs on cell proliferation activity. The proliferation activity of tumor cells is important in the invasion/metastasis of the tumor. Once we had identified the most effective GPC3-specific shRNA transfectants (MHCC97-H/GPC3shRNA1), we examined the cell proliferation activity of the transfected cells using the MTT assay. As shown in Fig. 3, the growth of MHCC97-H cells in vitro was markedly inhibited after the transfection of pGenesil-1-GPC3shRNA1 $(\mathrm{P}<0.05)$. This indicates a positive relation between the expression of GPC3 and the rate of hepatocellular cancer cell growth.

Effect of silencing GPC3 using shRNA on the colony formation potential of MHCC97-H cells. In order to document the ability of MHCC97-H/GPC3-shRNA1 to form colonies, single-cell suspensions were plated at a density of 100 cells in 30-mm culture dishes. After 12 days, MHCC97-H/GPC3shRNA1 cells, compared with MHCC97-H and MHCC97-H/ negative-shRNA cells, had a significant reduction in their ability to form colonies (Fig. 4A), and their ability to form colonies correlated with GPC3 expression $(\mathrm{P}<0.05)$ (Fig. 4B).

Effects of GPC3-specific shRNAs on the cell invasion activity. Invasion assays were also carried out using Matrigel-coated transwell culture chambers. After $24 \mathrm{~h}$, invading cells were counted with image analysis. MHCC97-H/GPC3-shRNA1 cells showed notable attenuated invasive activities as compared with MHCC97-H and MHCC97-H/negative-shRNA cells $(\mathrm{P}<0.05)$ (Fig. 5). These results demonstrate that the invasive ability of hepatocellular cells correlated with GPC3 expression, and GPC3 silencing alone is sufficient to attenuate invasion in hepatocellular carcinoma cells.

GPC3 gene silencing suppresses cell proliferation in vivo. The effect of GPC3 on in vivo tumor growth was assessed by the subcutaneous injection of MHCC97-H/GPC3-shRNA1 cells and MHCC97-H/negative-shRNA cells for 21 days. As 
shown in Fig. 6, a remarkable reduction of tumor size was observed in the MHCC97-H/GPC3-shRNA1 group as compared to that of the control group $(\mathrm{P}<0.05)$. By day 21 after cell injection, the average tumor weight $(n=4)$ of the MHCC97-H/ GPC3-shRNA1 and MHCC97-H/negative-shRNA groups was $1.31 \pm 0.06$ and $2.83 \pm 0.17 \mathrm{~g}$, respectively $(\mathrm{P}<0.01)$, indicating that knockdown of GPC3 in hepatocellular cancer cells reduced their tumorigenic potential.

\section{Discussion}

Hepatocellular carcinoma (HCC), a common primary cancer of liver origin, claims over 600,000 deaths globally per year with a rising incidence (14). Due to the impediment in its diagnosis and treatment caused by its high heterogeneity and the uncertainty concerning the concrete mechanism of HCC carcinogenesis, the prognosis of most patients is not satisfactory. In recent years, with the advancement of molecular inhibitors like sorafenib (15), sunitinib (16) and lapatinib (17), the outcomes of patients with advanced disease has improved tremendously, which intensifies the need for the development of potent molecular targets and clarifaction of the mechanism of HCC tumorgenesis.

Glypican-3 (GPC3), a newly identified candidate biomarker for HCC, is a glypican family member which characterized as an HSPGs anchored in the cellular surface with GPI anchors, encoding 8 exons and 7 introns. The transcription factor binding sites locating in the promoter region enable GPC3 to regulate cellular growth and organ development through spatial and temporal adjustment of various signal transduction pathways, such as IGF-II, Hh, BMP2, BMP and FGF-7 (8). A plethora of in vitro evidence has showed that the expression of GPC3 mRNA and protein were remarkably increased in the primary sites and serums of HCC patients, comparing normal people or patients with benign liver lesions. The expression of OCI-5, a rat homologue of GPC3 was found to gradually increase during the occurrence and progression of HCC in a rat model. Furthermore, data obtained from different studies (18-20) and in our laboratory (unpublished data) have revealed that GPC3 expression is correlated with such clinical features of HCC as tumor staging, differential degree and invasive ability. HCC patients with increased levels of GPC3 have relatively shorter survival rates than those with lower GPC3 expression, indicating that elevated levels of GPC3 could be an independent factor of poor prognosis in HCC (21). An extremely strong GPC3 overexpression has been closely related to the recurrence of HCC after surgery resection (our unpublished data). Thus, we hypothesized that GPC3 may play a role in promoting carcinogenesis and in the development of HCC, especially in the advanced stage.

In our study, MHCC97-H, a HCC cell line possessing high metastatic potential, were chosen as the RNA interfering subject, because it demonstrated increased levels of GPC3 as compared with other HCC cell lines, HepG2, SMMC-7721 and HCCL-M6. Since MHCC97-H is a highly invasive subtype of MHCC which were separated from a metastatic model of human HCC in nude mice $(22,23)$, our experiments could present a more vivid aspect concerning the function of GPC3 in the development of human HCC. The cytological results indicate a relationship between the declined GPC3 expression and the suppression of cell proliferation, and together with further in vivo experiments support our hypothesis that GPC3 may be a direct factor contributing to the carcinogenesis and development of HCC by controlling cellular proliferation. On the other hand, lower expression of GPC3 was also associated with a remarkable containment of the invasion of MHCC97-H cells in vitro. Our results are in accordance with previous studies that GPC3 may facilitate the development of HCC $(10,24,25)$. Moreover, it has been shown in vivo that overexpression of GPC3 in hepatocyte suppresses cellular proliferation and liver regeneration (26), suggesting a possible reason for the poorer outcomes observed in HCC patients overexpressing GPC3. Interestingly, our observation conflicts with the reports that knockdown of GPC3 in HepG2, Hep 3B hepatoma cells stimulates cellular growth of $\operatorname{HCC}(27,28)$. The application of different cell lines and intriguing functional mechanisms of GPC3 may explain the discrepancy and more animal models would help to circumvent the question.

Given the ability of GPC3 to perform disparate effects in different tissues, it was not surprising to find its distinct expressing and functioning pattern. Unlike HCC cells, GPC3 was down-regulated in ovarian cancer, mesothelioma, breast cancer, gastric cancer and lung cancer cells (29-34), implicating GPC3 as an inhibitor of tumor growth and progression. GPC3 re-expression induced impairment of the invasive capacities, motility and proliferation of mammary adenocarcinoma cells (35). Elevated levels of GPC3 were associated with cellular growth inhibition of ovarian clear cell carcinoma cells (11). The time- and tissue- specific expression may account for the peculiar function of GPC3 in HCC. Furthermore, pathways involved in the development and progression of HCC may not be part of the molecular mechanisms of other cancers. Previous studies have indicated that GPC3 in hepatoma cells may interact with IGF-II, Wnt, Hh, FGF and TGF- $\beta(23,25,36,37)$. A forthcoming investigation about its role in angiogenesis is warranted since progression of HCC typically exhibits active angiogenesis.

In summary, our findings provide evidence that GPC3 contributes to the progression of HCC through modulation of cell growth and invasion. The evidence presented herein only minimally adds to the current state of knowledge of the mechanisms of HCC. Further studies are needed to clarify the specific functional mechanism of GPC3 in HCC and its potential as a molecular target in the treatment of this disease. Moreover, the investigation of the possible role of GPC3 in other cancer types is warranted.

\section{Acknowledgements}

We gratefully thank Chengwei Lv from the Cancer Biotherapy center for the collection and maintenance of the HCC cell lines HepG2, SMMC-7721 and HCCL-M6 used in this study. Bin Chen is acknowledged for providing the HCC cell line MHCC97-H. Rong Li is acknowledged for excellent technical assistance. The project was supported by the National Natural Science Foundation of China (Grant No. 81001212), the China Postdoctoral Science Foundation (Grant No. 20080431074), the Foundation of Zhejiang Provincial Educational Committee (Grant No. Y201019175), and the Zhejiang Provincial Health Bureau Foundation (Grant No. 2010KYB036). 


\section{References}

1. Tung-Ping PR, Fan ST and Wong J: Risk factors, prevention, and management of postoperative recurrence after resection of hepatocellular carcinoma. Ann Surg 232: 10-24, 2000.

2. Capocaccia R, Sant M, Berrino F, Simonetti A, Santi V and Trevisani F: Hepatocellular carcinoma: trends of incidence and survival in Europe and the United States at the end of the 20th century. Am J Gastroenterol 102: 1660-1671, 2007.

3. Ng KK, Lo CM, Liu CL, Poon RT, Chan SC and Fan ST: Survival analysis of patients with transplantable recurrent hepatocellular carcinoma: implications for salvage liver transplant. Arch Surg 143: 68-74, 2008.

4. Li M, Choo B, Wong ZM, Filmus J and Buick RN: Expression of OCI-5/glypican 3 during intestinal morphogenesis: regulation by cell shape in intestinal epithelial cells. Exp Cell Res 235: 3-12, 1997.

5. Pellegrini M, Pilia G, Pantano S, et al: Gpc3 expression correlates with the phenotype of the Simpson-Golabi-Behmel syndrome. Dev Dyn 213: 431-439, 1998.

6. Pilia G, Hughes-Benzie RM, MacKenzie A, et al: Mutations in GPC3, a glypican gene, cause the Simpson-Golabi-Behmel overgrowth syndrome. Nat Genet 12: 241-247, 1996.

7. Cano-Gauci DF, Song HH, Yang H, et al: Glypican-3-deficient mice exhibit developmental overgrowth and some of the abnormalities typical of Simpson-Golabi-Behmel syndrome. J Cell Biol 146: 255-264, 1999.

8. Gonzalez AD, Kaya M, Shi W, Song H, Testa JR, Penn LZ and Filmus J: OCI-5/GPC3, a glypican encoded by a gene that is mutated in the Simpson-Golabi-Behmel overgrowth syndrome induces apoptosis in a cell line-specific manner. J Cell Biol 141: 1407-1414, 1998.

9. Peters MG, Farias E, Colombo L, Filmus J, Puricelli L and Bal Bal de Kier Joffé E: Inhibition of invasion and metastasis by glypican-3 in a syngeneic breast cancer model. Breast Cancer Res Treat 80: 221-232, 2003.

10. Capurro MI, Xiang YY, Lobe C and Filmus J: Glypican-3 promotes the growth of hepatocellular carcinoma by stimulating canonical Wnt signaling. Cancer Res 65: 6245-6254, 2005.

11. Sakurai M, Shibata K, Umezu T, et al: Growth-suppressing function of glypican-3 (GPC3) via insulin like growth factor II (IGF-II) signaling pathway in ovarian clear cell carcinoma cells Gynecol Oncol 119: 332-336, 2010.

12. Sung YK, Hwang SY,Park MK, et al: Glypican-3 is overexpressed in human hepatocellular carcinoma. Cancer Sci 94: 259-262, 2003.

13. Tuschl T: Expanding small RNA interference. Nat Biotechnol 20: 446-448, 2002.

14. Gomaa AI, Khan SA, Toledano MB, Waked I and TaylorRobinson SD: Hepatocellular carcinoma: epidemiology, risk factors and pathogenesis. World J Gastroenterol 14: 4300-4308, 2008.

15. Llovet JM, Ricci S, Mazzaferro V, et al: Sorafenib in advanced hepatocellular carcinoma. N Engl J Med 359: 378-390, 2008.

16. Zhu AX, Sahani DV, Duda DG, et al: Efficacy, safety, and potential biomarkers of sunitinib monotherapy in advanced hepatocellular carcinoma: a phase II study. J Clin Oncol 27: 3027-3035, 2009.

17. Bekaii-Saab T, Markowitz J, Prescott N, et al: A multiinstitutional phase II study of the efficacy and tolerability of lapatinib in patients with advanced hepatocellular carcinomas. Clin Cancer Res 15: 5895-5901, 2009.

18. Zhu ZW, Friess H, Wang L, et al: Enhanced glypican-3 expression differentiates the majority of hepatocellular carcinomas from benign hepatic disorders. Gut 48: 558-564, 2001
19. Yasuda E, Kumada T, Toyoda H, et al: Evaluation for clinical utility of GPC3, measured by a commercially available ELISA kit with Glypican-3 (GPC3) antibody, as a serological and histological marker for hepatocellular carcinoma. Hepatol Res 40: 477-485, 2010.

20. Hsu HC, Cheng W and Lai PL: Cloning and expression of a developmentally regulated transcript MXR7 in hepatocellular carcinoma: biological significance and temporospatial distribution. Cancer Res 57: 5179-5184, 1997.

21. Shirakawa H, Suzuki H, Shimomura M, et al: Glypican-3 expression is correlated with poor prognosis in hepatocellular carcinoma. Cancer Sci 100: 1403-1407, 2009.

22. Tian J, Tang ZY, Ye SL, Liu YK, Lin ZY, Chen J and Xue Q New human hepatocellular carcinoma (HCC) cell line with highly metastatic potential (MHCC97) and its expressions of the factors associated with metastasis. Br J Cancer 81: 814-821, 1999.

23. Li Y, Tang ZY, Ye SL, et al: Establishment of cell clones with different metastatic potential from the metastatic hepatocellular carcinoma cell line MHCC97. World J Gastroenterol 7: 630-636, 2001.

24. Cheng W, Tseng CJ, Lin TT, Cheng I, Pan HW, Hsu HC and Lee YM: Glypican-3-mediated oncogenesis involves the Insulinlike growth factor-signaling pathway. Carcinogenesis 29: 1319-1326, 2008

25. Filmus $\mathbf{J}$ and Capurro $M$ : The role of glypican-3 in the regulation of body size and cancer. Cell Cycle 7: 2787-2790, 2008.

26. Liu B, Bell AW, Paranjpe S, et al: Suppression of liver regeneration and hepatocyte proliferation in hepatocyte-targeted glypican 3 transgenic mice. Hepatology 52: 1060-1067, 2010.

27. Farooq M, Hwang SY, Park MK, Kim JC, Kim MK and Sung YK: Blocking endogenous glypican-3 expression releases Hep 3B cells from G1 arrest. Mol Cells 15: 356-360, 2003.

28. Sung YK, Hwang SY, Farooq M, Kim JC and Kim MK: Growth promotion of HepG2 hepatoma cells by antisense-mediated knockdown of glypican-3 is independent of insulin-like growth factor 2 signaling. Exp Mol Med 35: 257-262, 2003.

29. Lin H, Huber R, Schlessinger D and Morin PJ: Frequent silencing of the GPC3 gene in ovarian cancer cell lines. Cancer Res 59: 807-810, 1999 .

30. Murthy SS, Shen T, De Rienzo A, et al: Expression of GPC3, an $\mathrm{X}$-linked recessive overgrowth gene, is silenced in malignant mesothelioma. Oncogene 19: 410-416, 2000.

31. Xiang YY, Ladeda V and Filmus J: Glypican-3 expression is silenced in human breast cancer. Oncogene 20: 7408-7412, 2001.

32. Zhu Z, Friess H, Kleeff J, et al: Glypican-3 expression is markedly decreased in human gastric cancer, but not in esophageal cancer. Am J Surg 184: 78-83, 2002.

33. Powell CA, Xu G, Filmus J, Busch S, Brody JS and Rothman PB: Oligonucleotide microarray analysis of lung adenocarcinoma in smokers and nonsmokers identifies GPC3 as a potential lung tumor suppressor. Chest 121 (3 Suppl): S6-S7, 2002.

34. Kim H, Xu GL, Borczuk AC, et al: The heparan sulfate proteoglycan GPC3 is a potential lung tumor suppressor. Am J Respir Cell Mol Biol 29: 694-701, 2003.

35. Buchanan C, Stigliano I, Garay-Malpartida HM, et al: Glypican-3 reexpression regulates apoptosis in murine adenocarcinoma mammary cells modulating PI3K/Akt and p38MAPK signaling pathways. Breast Cancer Res Treat 119: 559-574, 2010.

36. Lai JP, Sandhu DS, Yu C, et al: Sulfatase 2 up-regulates glypican 3, promotes fibroblast growth factor signaling, and decreases survival in hepatocellular carcinoma. Hepatology 47: 1211-1222, 2008.

37. Midorikawa Y, Ishikawa S, Iwanari H, et al: Glypican-3, overexpressed in hepatocellular carcinoma, modulates FGF2 and BMP-7 signaling. Int J Cancer 103: 455-465, 2003. 